\title{
Rat Model for Poly-Autoimmunity
}

\author{
Maria Staykova \\ Neurosciences Research Unit, The Canberra Hospital and The John Curtin School of Medical Research, \\ The Australian National University, GPO Box 334, Canberra City, ACT 2600, Australia
}

Received 2012-09-03, Revised 2012-09-07; Accepted 2012-09-07

\begin{abstract}
Around $30 \%$ of the male PVG rats have very low testosterone levels. Upon immunisation with myelin basic protein in complete Freund's adjuvant they develop experimental autoimmune encephalomyelitis and adjuvant arthritis. The androgen insensitive syndrome in these rats could be used in studies on poly-autoimmunity as well as in studies on variations in the sexual differentiation of brain and behavior.
\end{abstract}

Keywords: Male PVG Rat, Experimental Autoimmune Encephalomyelitis, Adjuvant Arthritis, PolyAutoimmunity, Sexual Behavior

\section{INTRODUCTION}

There is vast literature on neuro-immuno-endocrynology with excellent reviews on multiple-autoimmunity, i.e., the existence of several autoimmune diseases (Bergsteinsdottir et al., 2000; Rojas-Villarraga et al., 2012; NNE, 2012). The numerous studies describing different parts in the hypothalamic-pituitary-adrenal axis and the interplay between the gonadal steroids and the immune system, with respect to autoimmunity in the central nervous system, have been critically reviwed (Mason et al., 1990; Whitacre, 2001; Arnaud, 2009; ZandmanGoddard et al., 2012; Zhang et al., 2012). The experimental models used for this research included adrenalectomy, gonadectomy or delivery of exogenous hormones (Bonthuis et al., 2010).

While studying the role of Nitric Oxide (NO) in the development of The Experimental Autoimmune Encephalomyelitis (EAE) in the DA, Lew, PVG and BN rat (Willenborg et al., 1999; 2007; Staykova et al., 2008 ), it became clear that about $30 \%$ of the male PVG rats develop not only EAE but also Adjuvant Arthritis (AA). (In 18 years of experimental work, in only seven male Lewis rats, did I observe swelling of the ankles after active induction of EAE).

The aim of this brief report is to point to the naturally occurring gonadal steroids' disbalance in a high percentage of the male PVG rats, which could be used in studies on poly-autoimmunity as well as in studies on sexual differentiation of brain and behavior.

\section{MATERIALS AND METHODS}

Male PVG rats, 10-20 week old, were bled via the lateral tail vein between 12 and 12.30 noon and immunized in both hind footpads with a total of $200 \mu \mathrm{L}$ emulsion containing $30 \mu \mathrm{g}$ guinea pig myelin basic protein and complete Freund's adjuvant (CFA, $4 \mathrm{mg}$ $\mathrm{ml}^{-1}$ Mycobacterium butyricum in IFA, Difco Laboratories, Detroit, USA). The progesterone and testosterone serum levels were tested with the routine methods in ACT Pathology, Canberra. Based on the testosterone levels, two groups were formed: one with normal values (control, $\mathrm{n}=4$ ) and one with levels below the sensitivity of the method $\left(0.69 \mathrm{nM} \mathrm{L} \mathrm{L}^{-1}\right)$ (experimental, $\mathrm{n}=6$ ). EAE clinical scores were assigned according to the accepted scale from no signs (score 0) to difficulty righting (score 3) (Staykova et al., 2002). Around day 13 after the immunisation, the animals in the control group started showing the typical neurologic signs of EAE that progressed from flaccid distal part of the tail (score 1) to entire tale flaccid (score 2). In the experimental group the paws and the ankles of all six rats started swelling eight-ten days after the immunisation and made difficult the evaluation of EAE. Swelling of hindpaws was quantified by measuring the thickness of the ankle from medial to lateral malleous with a constant tension caliper. On day 15 all animals were euthanized and the lumbar spinal cords and ankles were fixed in formalin. After decalcification, the joints were processed by the routine paraffin method. Paraffin 
sections from both types of specimens (spinal cord and joints) were stained with haematoxylin-eosin. All animals received care that met the standards of the Animal Ethics Committee of the Australian National Health and Medical Research Council.

\section{RESULTS AND DISCUSSION}

The histopathologic picture of the lumbar spinal cords taken from male PVG rats, with normal testosterone levels or with testosterone levels below the sensitivity of the test (Fig. 1 table), was the same-with meningeal mononuclear cell inflammation (Fig. 1A and C). Thus, both groups developed comparable EAE.

In contrast, the mean ankle thickness increased from $6.8 \pm 0.2 \mathrm{~mm}$ (before immunization) to $12.4 \pm 0.9 \mathrm{~mm}$ (on day 15) in the group with low testosterone levels and there was a clear difference in the histology of the joints - normal for the control group and arthritic for the experimental group (Fig. 1B and D). This seems not to be dependent on the age of the animals in the experimental group although the numbers are too small for conclusions. In all AA rats there was a strong involvement of polymorph nuclear cells (Fig. 1E) that is typical for inflammatory cartilage destruction in rat adjuvant arthritis and was also reported for patients with rheumatoid arthritis (Glenn et al., 1977; Mohr et al., 1981; Eden et al., 2001; Ribbhammar et al., 2003; Hutsona and Hasthorpe, 2005).

Different rat strains have different sensitivity to $\mathrm{T}$ cell mediated autoimmunity (Swanborg, 2001). In EAE (actively induced with myelin basic protein in CFA) the order for the males is $\mathrm{DA}=$ Lew $>$ PVG $>$ $\mathrm{BN}$ (Willenborg et al., 2007). With respect to AA, the male DA rat is also most susceptible and the condition could be induced even with incomplete Freund's adjuvant (Mason et al., 1990; Ribbhammar et al., 2003). In the studies on AA in male Lew rat were used complete Freund's adjuvants with either Mycobacterium tuberculosis (Kong et al., 1999) or $M$. butyricum (Billiau and Matthys, 2001).

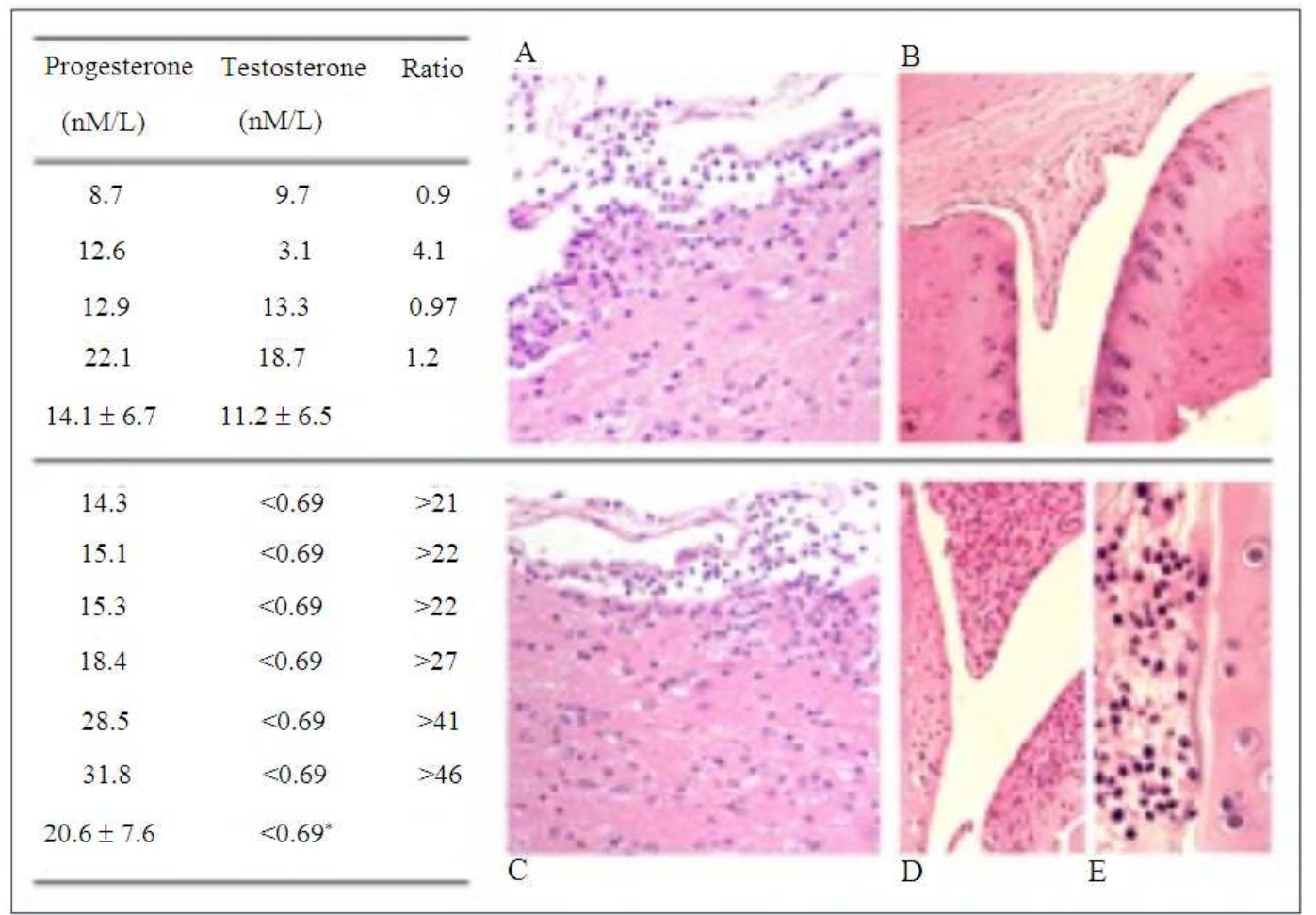

Fig. 1. Male PVG rat levels for progesterone and testosterone and histology of spinal cords and joints. Table: individual values of gonadal hormones in the control and experimental group. The pictures for haematoxylin-eosin stained lumbar spinal cord (A $=$ control group; $\mathrm{C}=$ experimental group $)$ and ankle joint $(\mathrm{B}=$ control group; $\mathrm{D}$ and $\mathrm{E}=$ experimental group $)$ are representative for the individual animals in each of the two groups. Original magnification $\times 40(A, B, C, D)$ and $\times 100(E)$ 


\section{CONCLUSION}

When the topic is poly-autoimmunity or multiple autoimmune syndrome, i.e., co-existence of more than two autoimmune diseases, than genetic analysis of the affected families helps to assess the risk (Ribbhammar et al., 2003; Criswell et al., 2005; Perez-Fernandez et al., 2012). On the other hand, models that reproduce autoimmune conditions (or phases of these conditions) are needed for experimental research.

Here we provide one more proof for the role of the endocrine system as a contributory cause in determining the susceptibility to two autoimmune conditions-EAE and AA. The fact that a good percentage of the male PVG rats have the androgen insensitive syndrome (previously called "testicular feminization syndrome", Hutsona and Hasthorpe, 2005) can be used in studies on poly-autoimmunity as well as in studies on variations in the sexual differentiation of brain and behavior (Bonthuis et al., 2010; Zuloaga et al., 2011).

\section{ACKNOWLEDGMENT}

This study was supported by grants from the Multiple Sclerosis Australia and the Canberra Hospital Private Practice Fund.

The author thanks sincerely Dr David O. Willenborg for his support, ACT Pathology for testing the samples for progesterone and testosterone and Anne Prins for the histology of spinal cords and joints.

Maria Staykova is a Visiting Fellow at the John Curtin School of Medical Research, Australian National University, Canberra.

\section{REFERENCES}

Arnaud, N., 2009. Gender and sex hormones in multiple sclerosis pathology and therapy. Frontiers Biosci., 14: 4477-4515. DOI: 10.2741/3543

Bergsteinsdottir, K., H.T. Yang, U. Pettersson and R. Holmdahl, 2000. Evidence for common autoimmune disease genes controlling onset, severity and chronicity based on experimental models for multiple sclerosis and rheumatoid arthritis. J. Immunol., 164: 1564-1568. PMID 10640775

Billiau, A. and P. Matthys, 2001. Modes of action of Freund's adjuvants in experimental models of autoimmune diseases. J. Leukoc. Biol., 70: 849-60. PMID: 11739546
Bonthuis, P., K. Cox, B. Searcy, P. Kumar and S. Tobet et al., 2010. Of mice and rats: Key species variations in the sexual differentiation of brain and behavior. Frontiers Neuroendocrinol., 31: 341-358. DOI: 10.1016/j.yfrne.2010.05.001

Criswell, L., K. Pfeiffer, R. Lum, B. Gonzales and J. Novitzke et al., 2005. Analysis of families in the Multiple Autoimmune Disease Genetics Consortium (MADGC) collection: The PTPN22 620W allele associates with multiple autoimmune phenotypes. Am. J. Hum. Genet., 76: 561-571. PMID: 15719322

Eden, W.V., J.P. Wagenaar-Hilbers and M.H. Wauben, 2001. Adjuvant arthritis in the rat. Curr. Protoc. Immunol. PMID: 18432736

Glenn, E.M., B.J. Bowman, N.A. Rohloff and R.J. Seely, 1977. A major contributory cause of arthritis in adjuvant-inoculated rats: Granulocytes. Agents Actions, 7: 265-282. PMID: 899976

Hutsona, J.M. and S. Hasthorpe, 2005. Testicular descent and cryptorchidism: The state of the art in 2004. J. Pediatric Surgery, 40: 297-302. DOI: 10.1016/j.jpedsurg.2004.10.033

Kong, Y.Y., U. Feige, I. Sarosi, B. Bolon and A. Tafuri et al., 1999. Activated T cells regulate bone loss and joint destruction in adjuvant arthritis through osteoprotegerin ligand. Nature, 402: 304-309. PMID: 10580503

Mason, D., I. MacPhee and F. Antony, 1990. The role of the neuroendocrine system in determining genetic susceptibility to experimental allergic encephalomyelitis in the rat. Immunology, 70: 1-5. PMID: 2354853

Mohr, W., A. Wild and H.P. Wolf, 1981. Role of polymorphs in inflammatory cartilage destruction in adjuvant arthritis of rats. Ann. Rheum. Dis., 40: 171-176. PMID: 7224688

NNE, 2012. Focus on neuro-immune interactions. Nature Neurosci. DOI: 10.1038/nn0812-1055

Perez-Fernandez, O.M., R.D. Mantilla, P. Cruz-Tapias, A. Rodriguez-Rodriguez and A. Rojas-Villarraga et al., 2012. Spondyloarthropathies in Autoimmune Diseases and Vice Versa. Autoimmune Dis., 2012: 1-7. DOI: 10.1155/2012/736384

Ribbhammar, U., L. Flornes, L. Backdahl, H. Luthman and S. Fossum et al., 2003. High resolution mapping of an arthritis susceptibility locus on rat chromosome 4 and characterization of regulated phenotypes. Hum. Mol. Genet., 12: 2087-2096. DOI: $10.1093 / \mathrm{hmg} / \mathrm{ddg} 224$ 
Rojas-Villarraga, A., J. Amaya-Amaya, A. RodriguezRodriguez, R.D. Mantilla and J.M. Anaya, 2012. Introducing polyautoimmunity: Secondary autoimmune diseases no longer exist. Autoimmune Dis., 2012: 1-9. DOI: 10.1155/2012/254319

Staykova, M.A., D. Linares, S.A. Fordham, J.T. Paridaen and D.O. Willenborg, 2008. The innate immune response to adjuvants dictates the adaptive immune response to autoantigens. J. Neuropathol. Exp. Neurol., 67: 543-554. DOI: 10.1097/NEN.0b013e31817713cc

Staykova, M.A., W. Cowden and D.O. Willenborg, 2002. Macrophages and nitric oxide as the possible cellular and molecular basis for strain and gender differences in susceptibility to autoimmune central nervous system inflammation. Immunol. Cell Biol., 80: 188-197. PMID: 11940120

Swanborg, R., 2001. Experimental autoimmune encephalomyelitis in the rat: Lessons in T-cell immunology and autoreactivity. Immunol. Rev., 184: $129-135$. DOI: $10.1034 /$ j.1600065x.2001.1840112.x

Whitacre, C.C., 2001. Sex differences in autoimmune disease. Nature Immunol., 2: 777-780. DOI: 10.1038/ni0901-777

Willenborg, D.O., M. Staykova, S. Fordham, N. O'Brien and D. Linares, 2007. The contribution of nitric oxide and interferon gamma to the regulation of the neuro-inflammation in experimental autoimmune encephalomyelitis. J. Neuroimmunol., 191: 16-25. PMID: 17904645
Willenborg, D.O., M.A. Staykova and W.B. Cowden, 1999. Our shifting understanding of the role of nitric oxide in autoimmune encephalomyelitis: A review. J. Neuroimmunol., 100: 21-35. DOI: 10.1016/S0165-5728(99)00212-X

Zandman-Goddard, G., E. Peeva, Z. Rozman, I. Ben-Zvi and P. Langevitz et al., 2012. Sex And Gender Differences In Autoimmune Diseases. Sex Gender Aspects Clin. Med. DOI: 10.1007/978-0-85729832-4_8

Zhang, M.A., D. Rego, M. Moshkova, H. Kebir and A. Chruscinski et al., 2012. Peroxisome ProliferatorActivated Receptor (PPAR) $\alpha$ and $-\gamma$ regulate IFN $\gamma$ and IL-17A production by human $\mathrm{T}$ cells in a sexspecific way. PNAS, 109: 9505-9510. DOI: 10.1073/pnas.1118458109

Zuloaga, D.G., J.E. Poort, C.L. Jordan and S.M. Breedlove, 2011. Male rats with the testicular feminization mutation of the androgen receptor display elevated anxiety-related behavior and corticosterone response to mild stress. Horm. Behav., 60: 380-388. PMID: 21801726 